\title{
Viability of superoxide-containing radical pairs as magnetoreceptors
}

Cite as: J. Chem. Phys. 151, 225101 (2019); https://doi.org/10.1063/1.5129608

Submitted: 30 September 2019 . Accepted: 06 November 2019 . Published Online: 09 December 2019

(D) Thomas C. Player, and (D) P. J. Hore

\section{COLLECTIONS}

Paper published as part of the special topic on Spin ChemistrySPIN2019
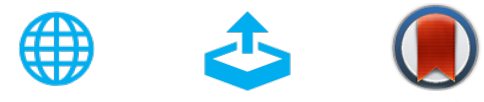

\section{ARTICLES YOU MAY BE INTERESTED IN}

On the optimal relative orientation of radicals in the cryptochrome magnetic compass

The Journal of Chemical Physics 151, 065103 (2019); https://doi.org/10.1063/1.5115445

Spin chemistry

The Journal of Chemical Physics 152, 120401 (2020); https://doi.org/10.1063/5.0006547

Nonspecific magnetic biological effects: A model assuming the spin-orbit coupling

The Journal of Chemical Physics 151, 204101 (2019); https://doi.org/10.1063/1.5127972

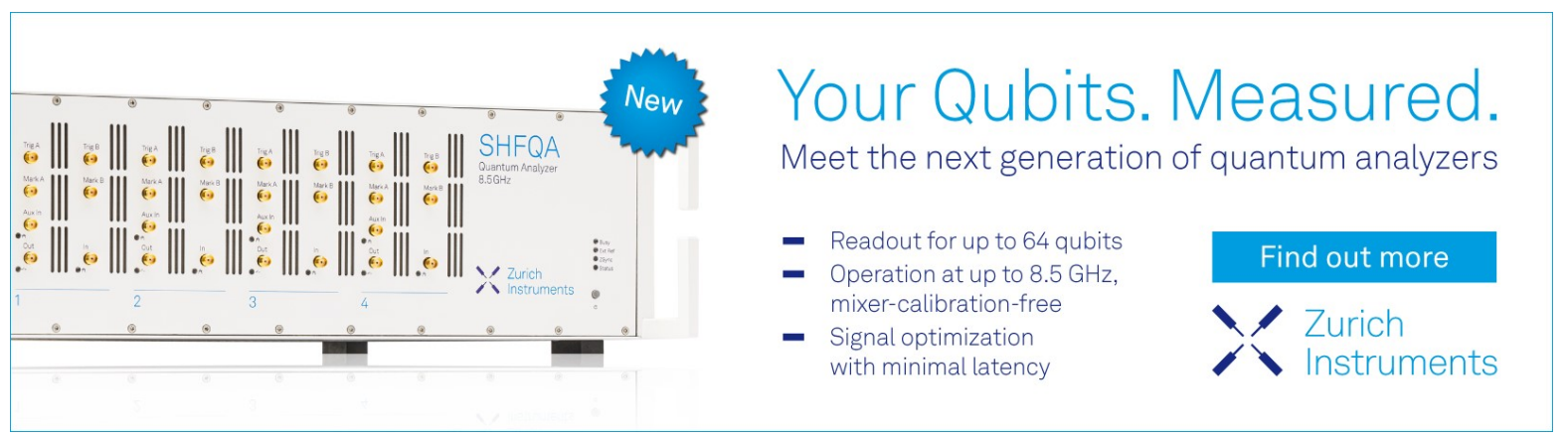




\title{
Viability of superoxide-containing radical pairs as magnetoreceptors
}

\author{
Cite as: J. Chem. Phys. 151, 225101 (2019); doi: 10.1063/1.5129608 \\ Submitted: 30 September 2019 • Accepted: 6 November 2019 • \\ Published Online: 9 December 2019
}

\author{
Thomas C. Player (iD) and P. J. Hore
}

\begin{abstract}
AFFILIATIONS
Physical and Theoretical Chemistry Laboratory, Department of Chemistry, University of Oxford, Oxford, United Kingdom
\end{abstract}

Note: The paper is part of the JCP Special Topic on Spin Chemistry.

a) Author to whom correspondence should be addressed: peter.hore@chem.ox.ac.uk

\begin{abstract}
The ability of night-migratory songbirds to sense the direction of the Earth's magnetic field is increasingly attributed to a photochemical mechanism in which the magnetic field acts on transient radical pairs in cryptochrome flavoproteins located in the birds' eyes. The magnetically sensitive species is commonly assumed to be $\left[\mathrm{FAD}^{\bullet-} \operatorname{TrpH}^{\bullet+}\right]$, formed by sequential light-induced intraprotein electron transfers from a chain of tryptophan residues to the flavin adenine dinucleotide chromophore. However, some evidence points to superoxide, $\mathrm{O}_{2}^{\bullet-}$, as an alternative partner for the flavin radical. The absence of hyperfine interactions in $\mathrm{O}_{2}^{\bullet-}$ could lead to a more sensitive magnetic compass, but only if the electron spin relaxation of the $\mathrm{O}_{2}^{\bullet-}$ radical is much slower than normally expected for a small mobile radical with an orbitally degenerate electronic ground state. In this study we use spin dynamics simulations to model the sensitivity of a flavin-superoxide radical pair to the direction of a $50 \mu \mathrm{T}$ magnetic field. By varying parameters that characterize the local environment and molecular dynamics of the radicals, we identify the highly restrictive conditions under which a $\mathrm{O}_{2}^{\bullet-}$-containing radical pair could form the basis of a geomagnetic compass sensor. We conclude that the involvement of superoxide in compass magnetoreception must remain highly speculative until further experimental evidence is forthcoming.
\end{abstract}

Published under license by AIP Publishing. https://doi.org/10.1063/1.5129608

\section{INTRODUCTION}

Night-migratory songbirds use a magnetic sense to navigate over long distances by detecting the direction of the Earth's $\sim 50 \mu \mathrm{T}$ magnetic field ${ }^{1-3}$ with a precision better than $5^{\circ}$., ${ }^{4,5}$ Two principal theories have been proposed to account for the operation of this compass: (1) a magnetite hypothesis, in which directional information comes from the interaction of the Earth's field with biogenic magnetic iron oxide crystals, ${ }^{6-8}$ and (2) a radical-pair mechanism, in which the quantum yields of light-dependent radical reactions vary with the orientation of the bird's head with respect to the geomagnetic field. ${ }^{9-11}$ The latter is currently the leading hypothesis and is our concern here. We focus exclusively on cryptochrome, a blue-light photoreceptor protein, as the most likely magnetic sensor. $^{11}$

\section{A. Conventional mechanism (M1)}

The conventional radical pair scheme (referred to here as M1), involving light-induced reactions in cryptochrome, is shown in the upper part of Fig. 1. Blue-light irradiation of purified cryptochromes forms the spin-correlated radical pair [FAD ${ }^{\bullet-} \mathrm{TrpH}^{\bullet+}$ ] (denoted RP1) by sequential electron transfers along a chain of three or four tryptophan ( $\operatorname{TrpH})$ residues to the noncovalently bound flavin adenine dinucleotide (FAD) cofactor. ${ }^{16-18}$ Only the singlet state of RP1 can return to the ground state, while the $\mathrm{TrpH}^{\bullet+}$ radical in both singlet and triplet states can deprotonate to form the longer lived and magnetically insensitive species, $\mathrm{FAD}^{\bullet-}+\operatorname{Trp}^{\bullet} .{ }^{19,20}$ The extent and timing of singlet-triplet interconversion in RP1 is believed to be influenced by the direction of the Earth's magnetic field such that the quantum yields of $\mathrm{FAD}^{\bullet-}$ and downstream signaling states depend on the bird's head direction with respect to the magnetic field axis. Changes in the yield of the signaling state that arise from the anisotropy of the magnetic interactions in RP1 are presumed to be the source of the directional information sent from sensors in the eyes to the brain. ${ }^{9,21}$ The operation of this mechanism under the dim light conditions experienced by nocturnal migrants has been discussed by Hiscock et al. ${ }^{22}$ 


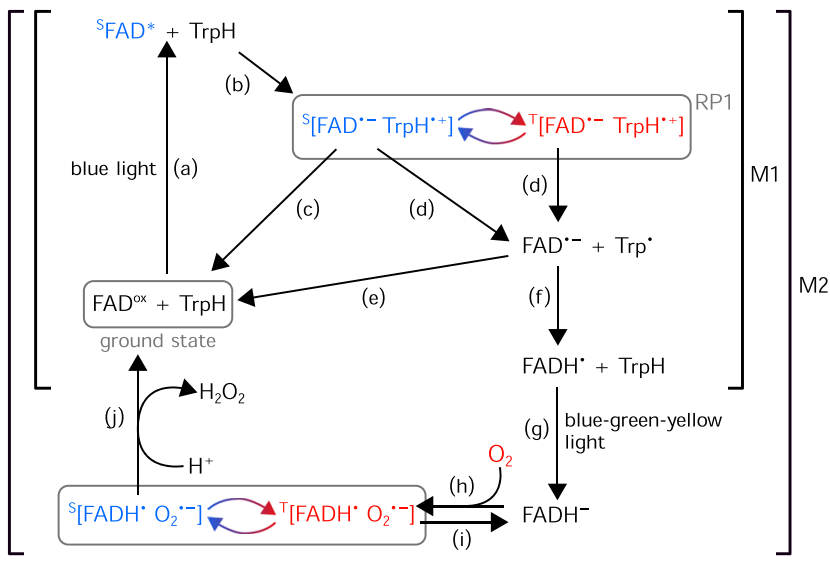

FIG. 1. The upper half of the figure shows the conventional scheme, M1, which accounts for observed magnetic field effects on the quantum yields of photochemical reactions in purified cryptochromes. ${ }^{20,23}$ (a) ${ }^{S} F A D^{*}$, the excited singlet state of $F A D$, is formed within the protein by excitation of the fully oxidized state, $F A D^{0 x}$ by blue light $(<500 \mathrm{~nm})$. (b) This leads to the creation of a radical pair (RP1) via sequential electron transfers along a chain of tryptophan $(\mathrm{TrpH})$ residues. RP1 is magnetically sensitive; the efficiency of its singlet-triplet interconversion (blue-red arrows) depends on the direction of the Earth's magnetic field. Only the singlet form of RP1 can recombine (c) to form the ground state, while the $\mathrm{TrpH}^{\bullet+}$ radical can lose a proton (d) to form a stabilized, magnetically insensitive radical pair, which then either recombines (e) to regenerate $F A D^{0 x}$ or goes on (f) to form the FADH ${ }^{\bullet}$ radical and TrpH. M1 can be extended to include reoxidation of the reduced flavin by $\mathrm{O}_{2}$ (superoxide mechanism, M2). $\mathrm{FADH}^{\bullet}$ can absorb a second photon $(<650 \mathrm{~nm})$, leading to the fully reduced flavin anion $\mathrm{FADH}^{-}(\mathrm{g})$. Reversible, spin-selective oxidation of $\mathrm{FADH}^{-}$by (triplet) $\mathrm{O}_{2}[(\mathrm{~h})$ and (i)] forms a [FADH $\mathrm{O}_{2}^{\bullet-}$ ] radical pair, initially in a triplet state. [FADH ${ }^{\bullet} \mathrm{O}_{2}^{\bullet-}$ ] can also undergo triplet-singlet interconversion, and only the singlet state can go on to form the ground state (j). In principle, $\left[\mathrm{FADH}^{\bullet} \mathrm{O}_{2}^{\bullet-}\right.$ ] can be magnetically sensitive. ${ }^{9,24-27}$ Electronic singlet and triplet states are shown in blue and red, respectively.

\section{B. Superoxide mechanism (M2)}

Although commanding a degree of support, ${ }^{9} \mathrm{M} 1$ is not the only conceivable cryptochrome-based model. A behavioral study in 2009 reported that European robins were disoriented by low-intensity magnetic fields oscillating at the Larmor frequency $(\sim 1.4 \mathrm{MHz})$ of a free electron spin in the Earth's magnetic field. ${ }^{11}$ Fields of similar strength at other radiofrequencies had a much smaller effect on the birds' ability to use their magnetic compass. Theoretical arguments suggested that resonance effects at the Larmor frequency could only be expected if one of the radicals in the pair were devoid of internal magnetic interactions.

This condition is clearly not met by either of the radicals that comprise RP1, both of which contain 10 magnetic nuclei (hydrogen and nitrogen) with significant hyperfine interactions. ${ }^{28}$ Nor is the condition satisfied by most other organic radicals, which typically have at least one ${ }^{1} \mathrm{H}$ or ${ }^{14} \mathrm{~N}$ atom in the neighborhood of the semioccupied molecular orbital. The only chemically and biologically plausible radical that meets the criterion of negligible hyperfine interactions seems to be superoxide, $\mathrm{O}_{2}^{\bullet-}, 11,24,26,28$ the reduced form of dioxygen $\left({ }^{16} \mathrm{O}\right.$ is $>99.7 \%$ naturally abundant and has no nuclear spin). However, the evidence for a radical containing a magnetically isolated electron spin has been considerably weakened by subsequent behavioral tests, performed under more rigorous conditions, which failed to reproduce a specific response at the Larmor frequency. ${ }^{29}$ This study, and one other, ${ }^{30}$ found that European robins are more effectively disoriented by weak broadband radiofrequency fields, an observation that would be consistent with $\left[\mathrm{FAD}^{\bullet-} \operatorname{TrpH}^{\bullet+}\right]$ as the sensor. ${ }^{3}$

Although the involvement of $\mathrm{O}_{2}^{\bullet-}$ is far from established, it cannot be dismissed. Figure 1 includes a mechanism (M2) by which a $\left[\mathrm{FADH}^{\bullet} \mathrm{O}_{2}^{\bullet-}\right.$ ] radical pair could arise in cryptochrome and, in principle, generate magnetic field effects. $\mathrm{O}_{2}$ efficiently oxidizes reduced flavins, both in vitro and in vivo ${ }^{26,32,33}$ and could form $\left[\mathrm{FADH}^{\bullet} \mathrm{O}_{2}^{\bullet-}\right]$ in a spin-correlated triplet state by reversible electron transfer from the fully reduced flavin, $\mathrm{FADH}^{-}$. A second electron transfer, within the singlet state of $\left[\mathrm{FADH}^{\bullet} \mathrm{O}_{2}^{\bullet-}\right.$ ], would return the protein to the ground state, $\mathrm{FAD}^{\mathrm{ox}}{ }^{24}$ Prior to these "dark reactions," light-dependent processes would be required to produce $\mathrm{FADH}^{-}$in a two-step process: first $\mathrm{FAD}^{\mathrm{ox}} \rightarrow \mathrm{FADH}^{\bullet}$ using blue light $(<500 \mathrm{~nm})$, as in the M1 scheme, and then $\mathrm{FADH}^{\bullet} \rightarrow \mathrm{FADH}^{-}$ with blue, green, or yellow light $(<650 \mathrm{~nm})$. Wavelengths longer than $500 \mathrm{~nm}$ are not strongly absorbed by $\mathrm{FAD}^{\mathrm{ox}}$ and so cannot on their own lead to $\mathrm{FADH}^{\bullet}$ or $\mathrm{FADH}^{-}$.

The evidence for an M2 mechanism rests largely on this difference in the absorption wavelengths of $\mathrm{FAD}^{\mathrm{ox}}$ and $\mathrm{FADH}^{\bullet}$. Immunohistochemical studies found that $\leq 600 \mathrm{~nm}$ light activated Cryla in chicken retinas but only when the birds had previously experienced light of shorter wavelengths $(<500 \mathrm{~nm})$, which could have converted the cryptochromes in their eyes into the $\mathrm{FADH}^{\bullet}$ state. $^{34-36}$ It has also been claimed that robins that had previously been exposed to white light could orient magnetically when a $50 \mu \mathrm{T}$ magnetic field and green $(\sim 565 \mathrm{~nm})$ or turquoise $(\sim 500 \mathrm{~nm})$ light were applied as nonoverlapping $\sim 100 \mathrm{~ms}$ pulses. ${ }^{37}$ Having a lifetime of microseconds at most, RP1 would not persist in the dark periods and so, it was argued, could not be the magnetic sensor. ${ }^{38}$ Although the biological parallels with migratory birds are unclear, similar studies on plants have also reported "dark" cryptochrome-related magnetic field effects. ${ }^{39}$ All of these observations have been attributed to an $\mathrm{M} 2$ mechanism involving [FADH ${ }^{\bullet} \mathrm{O}_{2}^{\bullet-}$ ] radical pairs. None of them has yet been independently replicated.

Theoretical considerations also point to flavin-containing radical pairs in which the counter radical has no or few hyperfine interactions. Spin dynamics simulations suggest that, other things being equal, the more asymmetric the distribution of hyperfine couplings between the two radicals, the larger the anisotropic magnetic field effects. ${ }^{28,40,41} \mathrm{~A}\left[\mathrm{FADH}^{\bullet} \mathrm{Z}^{\bullet}\right.$ ] pair in which $\mathrm{Z}^{\bullet}$ has no hyperfine interactions (e.g., $\mathrm{O}_{2}^{\bullet-}$ ) could be up to 100 times more sensitive to weak magnetic fields than $\left[\mathrm{FAD}^{\bullet-} \operatorname{TrpH}^{\bullet+}\right]^{28}$

\section{C. $\mathrm{O}_{2}^{--}$spin relaxation}

The main theoretical objection to an M2 mechanism is that spin relaxation, the process that destroys spin coherence in a radical pair, is expected to be unusually fast for $\mathrm{O}_{2}^{\bullet-24,42}$ If the electron spins in a radical pair relax before the magnetic field has time to affect the singlet-triplet interconversion, then there can be no magnetic field effect on the reaction yield. Several theoretical studies have assessed the spin relaxation of RP1, focusing mainly on motional modulation of hyperfine interactions. ${ }^{43-45}$ Three main conclusions 
emerged: (a) Radical pairs with spin relaxation faster than $\sim 1 \mu$ s do not show strong sensitivity to a $\sim 50 \mu \mathrm{T}$ magnetic field. (b) $\mathrm{TrpH}^{\bullet+}$ is less likely than $\mathrm{FAD}^{\bullet-}$ to spin-relax slowly enough to be compatible with geomagnetic sensing. (c) If the dominant relaxation process arises from fluctuations in hyperfine interactions, then a radical with fewer hyperfine interactions than $\operatorname{TrpH}^{\bullet+}$ (ideally none at all) would probably have slower spin relaxation and so give rise to larger magnetic field effects, other things being equal. Superficially, (b) and (c) could be taken as support for a $\left[\mathrm{FADH}^{\bullet} \mathrm{O}_{2}^{\bullet-}\right.$ ] sensor.

However, modulation of hyperfine interactions is not the only route by which electron spins relax. In the gas phase, $\mathrm{O}_{2}^{--}$has an orbitally degenerate electronic ground state $\left({ }^{2} \Pi_{\mathrm{g}}\right)$ and a significant spin-orbit coupling $\left(\lambda=-160 \mathrm{~cm}^{-1}\right.$, equivalent to $170 \mathrm{~T}$ or $0.74 k_{\mathrm{B}} T$ at $310 \mathrm{~K}$ ). This means that the electron spin angular momentum in a free $\mathrm{O}_{2}^{\bullet-}$ radical in solution is principally quantized along the molecular axis and consequently relaxes on the same time scale as molecular rotation, i.e., well within a nanosecond, preventing a $50 \mu \mathrm{T}$ magnetic field having any significant effect on the yield of the reaction products. ${ }^{47}$ Such rapid spin relaxation explains why the electron paramagnetic resonance (EPR) spectrum of $\mathrm{O}_{2}^{\bullet-}$ in a liquid has never been reported, and why unambiguous magnetic field effects on $\mathrm{O}_{2}^{\bullet-}$-containing radical pairs have only been observed at field strengths orders of magnitude stronger than $50 \mu \mathrm{T} .^{42,48}$ The vast majority of organic radicals do not suffer in this way because they have lower symmetry than $\mathrm{O}_{2}^{\bullet-}$, with the consequence that orbital angular momentum is quenched, greatly reducing the effects of spin-orbit coupling. The only obvious situation in which $\mathrm{O}_{2}^{\bullet-}$ could be part of a geomagnetic sensor would be in an asymmetric environment that both removed the degeneracy of its highest occupied $\left(\pi_{\mathrm{g}}\right)$ molecular orbitals and prevented rapid molecular reorientation. Such inhibition of spin relaxation has allowed EPR spectra of $\mathrm{O}_{2}^{\bullet-}$ to be observed in frozen solutions ${ }^{49}$ and alkali halide crystals. ${ }^{50}$ Most of the proponents of an M2-type mechanism have ignored or casually dismissed the rapid spin relaxation of $\mathrm{O}_{2}^{\bullet-35-37,51,52}$

The only other way in which the problem of rapid spin relaxation in $\mathrm{O}_{2}^{\circ-}$ might be avoided would be if Kattnig's radicalscavenging mechanism ${ }^{47,53}$ turns out to be operative in cryptochrome. Consideration of this possibility, for which there is currently no experimental evidence, is beyond the scope of this report.

The aim of the present study is to determine the conditions under which spin relaxation might be slow enough $(\sim 1 \mu \mathrm{s})$ to make
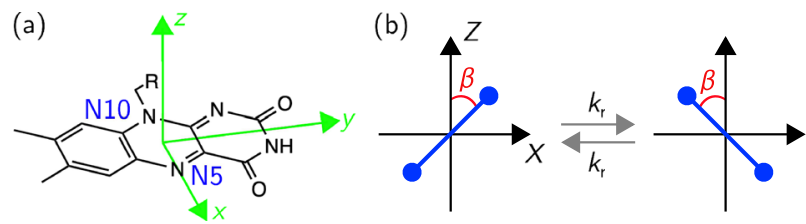

FIG. 2. (a) Structure of FAD and the molecular axis system $(x, y, z)$ of FADH (b) Representation of the two-site rocking motion of the $\mathrm{O}_{2}^{\bullet-}$ radical (blue) in the space-fixed XZ-plane. In its average orientation, the molecular axis of $\mathrm{O}_{2}^{\bullet-}$ is parallel to the $Z$-direction. Three relative orientations of the two radicals are considered, with the $\mathrm{FADH}^{\bullet}$ radical rotated such that its $Z$-axis is parallel to the $X-, Y_{-}$, or Z-axis.
$\mathrm{O}_{2}^{\bullet-}$ a viable component of a geomagnetic compass sensor. To this end, we report spin dynamics simulations of $\left[\mathrm{FADH}^{\bullet} \mathrm{O}_{2}^{\bullet-}\right.$ ] in which the $\mathrm{O}_{2}^{\bullet-}$ undergoes a rocking motion (small-angle rotational jumps) in an asymmetric electronic environment that removes the degeneracy of its $\pi_{\mathrm{g}}$-orbitals (Fig. 2 ).

\section{THEORY}

The $\mathrm{FADH}^{\bullet}$ radical was modeled by means of a spin Hamiltonian containing the two largest hyperfine interactions-with nitrogen N5 and N10 (Fig. 2)-which dominate the anisotropic spin dynamics of the $\left[\mathrm{FADH}^{\bullet} \mathrm{Z}^{\bullet}\right]$ radical pair. ${ }^{28,54}$ The remaining hyperfine couplings in $\mathrm{FADH}^{\bullet}$, being either small or nearly isotropic, have a relatively minor influence on the anisotropic magnetic sensitivity ${ }^{28}$ and are ignored here. Both ${ }^{14} \mathrm{~N}$ hyperfine tensors are approximately axially symmetric around the flavin $z$-axis (the normal to the molecular plane, Fig. 2). ${ }^{55}$ The small rhombic parts of these interactions were neglected, giving principal components $(-0.0914,-0.0914$, and 1.4767$) \mathrm{mT}$ for N5 and $(0.00255,0.00255$, $0.74670) \mathrm{mT}$ for N10. Adding the electron Zeeman interaction with the external magnetic field $\boldsymbol{B}_{0}$, the spin Hamiltonian of $\mathrm{FADH}^{\bullet}$ becomes

$$
\hat{H}_{\mathrm{F}}=\boldsymbol{\omega} \cdot \hat{\boldsymbol{R}}+\sum_{i=1,2} \hat{\boldsymbol{R}} \cdot \mathbf{A}^{(i)} \cdot \hat{\boldsymbol{I}}^{(i)}
$$

$\hat{\boldsymbol{R}}$ and $\hat{\boldsymbol{I}}^{(i)}$ are the spin angular momentum operators of the electron and the two nitrogens, respectively, with $\hat{\boldsymbol{R}}=\left(\hat{R}_{X}, \hat{R}_{Y}, \hat{R}_{Z}\right)$ and similarly for $\hat{\boldsymbol{I}}^{(i)}$. The hyperfine tensors, $\mathbf{A}^{(i)}$, are diagonal, with elements given by the principal components listed above. Permutation of these elements served to orient $\mathrm{FADH}^{\bullet}$ with its $z$-axis parallel to either the $X, Y$, or $Z$ space-fixed axis (Fig. 2). Ignoring the small anisotropy of the Zeeman interaction, $\boldsymbol{\omega}=-\left(g \mu_{\mathrm{B}} / \hbar\right) \boldsymbol{B}_{0}$ $=-\gamma_{\mathrm{e}} \boldsymbol{B}_{0}$ with $g \approx 2$. Throughout, we use dimensionless spin operators and write the spin Hamiltonians in angular frequency units. Spin relaxation in $\mathrm{FADH}^{\bullet}$ is assumed to be negligibly slow.

Turning to $\mathrm{O}_{2}^{\bullet-}$, the important internal interactions are the spin-orbit coupling $\left(\hat{H}_{\mathrm{SOC}}\right)$ and ligand field splitting $\left(\hat{H}_{\mathrm{LFS}}\right)$, which have the following nonzero matrix elements:

$$
\begin{gathered}
\left\langle m_{\mathrm{S}}, \pi_{ \pm}\left|\hat{H}_{\mathrm{SOC}}\right| m_{\mathrm{S}}, \pi_{ \pm}\right\rangle= \pm m_{\mathrm{S}} \lambda, \\
\left\langle m_{\mathrm{S}}, \pi_{x}\left|\hat{H}_{\mathrm{LFS}}\right| m_{\mathrm{S}}, \pi_{x}\right\rangle=-\left\langle m_{\mathrm{S}}, \pi_{y}\left|\hat{H}_{\mathrm{LFS}}\right| m_{\mathrm{S}}, \pi_{y}\right\rangle=\frac{1}{2} \Delta,
\end{gathered}
$$

where $\left|\pi_{ \pm}\right\rangle$are linear combinations of the two antibonding $\pi$ orbitals, $\left|\pi_{ \pm}\right\rangle=\left(\left|\pi_{x}\right\rangle \pm \mathrm{i}\left|\pi_{y}\right\rangle\right) / \sqrt{2}$, and $m_{\mathrm{S}}= \pm \frac{1}{2}$ is the magnetic quantum number of the electron spin. $\lambda$ and $\Delta$ are the strengths of the two interactions. It is convenient to rewrite Eq. (2) using a fictitious spin- $\frac{1}{2}$ operator, $\hat{\boldsymbol{L}}$, to represent the orbital angular momentum. When the molecular axis of $\mathrm{O}_{2}^{\bullet-}$ is parallel to the $Z$-axis (Fig. 2),

$$
\hat{H}_{\mathrm{SOC}}+\hat{H}_{\mathrm{LFS}}=2 \lambda \hat{S}_{Z} \hat{L}_{Z}+\Delta \hat{L}_{X},
$$

where $\hat{S}$ is the electron spin operator and a factor of 2 has been included in both terms because the angular momentum quantum number for the orbital motion $(l=1)$ is twice that of a spin- $\frac{1}{2}$ particle. 
The Zeeman interaction of $\mathrm{O}_{2}^{\bullet-}$ is given by

$$
\hat{H}_{\mathrm{Z}}=-\frac{g_{\mathrm{S}} \mu_{\mathrm{B}}}{\hbar} \boldsymbol{B} \cdot \hat{\boldsymbol{S}}-\frac{2 g_{\mathrm{L}} \mu_{\mathrm{B}}}{\hbar} \boldsymbol{B} \cdot \hat{\boldsymbol{L}}=\boldsymbol{\omega} \cdot(\hat{\boldsymbol{S}}+\hat{\boldsymbol{L}}) .
$$

The factor of 2 in the $\boldsymbol{B} \cdot \hat{\boldsymbol{L}}$ term again accounts for the orbital angular momentum quantum number. The $g$-values were taken as $g_{S}=2$ and $g_{\mathrm{L}}=1$.

Spin relaxation in $\mathrm{O}_{2}^{--}$was introduced by allowing the radical to jump between two orientations rotated around the $Y$-axis by angles $\pm \beta$ (Fig. 2). This rocking motion was modeled as a symmetric two-site exchange process with a first-order rate constant $k_{\mathrm{r}}$. The total spin-orbital Hamiltonian for $\mathrm{O}_{2}^{\bullet-}$, therefore, becomes

$$
\begin{aligned}
\hat{H}_{\mathrm{O}_{2}}( \pm \beta)= & 2 \lambda\left(\hat{L}_{Z} \cos \beta \pm \hat{L}_{X} \sin \beta\right)\left(\hat{S}_{Z} \cos \beta \pm \hat{S}_{X} \sin \beta\right) \\
& +\Delta\left(\hat{L}_{X} \cos \beta \mp \hat{L}_{Z} \sin \beta\right)+\boldsymbol{\omega} \cdot(\hat{\boldsymbol{S}}+\hat{\boldsymbol{L}}) .
\end{aligned}
$$

As in comparable treatments, ${ }^{28,31,44,55}$ singlet and triplet radical pairs were assumed to react independently with the same rate constant, $k=10^{6} \mathrm{~s}^{-1}$, giving a radical pair lifetime of $1 \mu \mathrm{s}$, a value that is probably near-optimal for sensing an Earth-strength magnetic field. ${ }^{57}$ The reactions modeled (Fig. 1) are ${ }^{\mathrm{S}}\left[\mathrm{FADH}^{\bullet} \mathrm{O}_{2}^{\bullet-}\right] \rightarrow \mathrm{FAD}^{\mathrm{ox}}+\mathrm{H}_{2} \mathrm{O}_{2}$ and ${ }^{\mathrm{T}}\left[\mathrm{FADH}^{\bullet} \mathrm{O}_{2}^{\bullet-}\right] \rightarrow \mathrm{FADH}^{-}+{ }^{3} \mathrm{O}_{2}$. Electron spin coupling of the two radicals was assumed to be negligible. The initial state of $\left[\mathrm{FADH}^{\bullet} \mathrm{O}_{2}^{\bullet-}\right.$ ] was a triplet (reflecting the ${ }^{3} \Sigma_{\mathrm{g}}^{-}$ground state of $\mathrm{O}_{2}$ ) with equal populations of the two $\mathrm{O}_{2}^{\bullet-}$ orientations. The dependence of the yield of the singlet product, $\Phi_{S}(\Omega)$, on the direction of the geomagnetic field, $\Omega$, was calculated using the approach described in Ref. 55 . The singlet yield anisotropy was quantified as $\Delta \Phi_{\mathrm{S}}=\max \left[\Phi_{\mathrm{S}}(\Omega)\right]-\min \left[\Phi_{\mathrm{S}}(\Omega)\right]$.

\section{RESULTS}

We calculated the anisotropy of the singlet yield, $\Delta \Phi_{S}$, to determine the conditions under which a $\left[\mathrm{FADH}^{\bullet} \mathrm{O}_{2}^{\bullet-}\right.$ ] radical pair could deliver significant information on the direction of an external $50 \mu \mathrm{T}$ magnetic field. Larger values of $\Delta \Phi_{S}$, resulting from slower spin relaxation, are assumed to provide a more efficient sensor and a more precise compass bearing.

Figure 3 shows the anisotropy of the magnetic field effect for three relative orientations of the radicals. In each case, $\Delta \Phi_{\mathrm{S}}$ is shown for three values of the ratio $\Delta / \lambda\left(0.1,1\right.$, and 10), with values of $k_{\mathrm{r}}$ spanning the range $10^{6}-10^{12} \mathrm{~s}^{-1}$, and $\beta=2^{\circ}$ and $5^{\circ}$. Strong dependence of the reaction yield on the magnetic field direction is seen when the ligand field splitting exceeds the spin-orbit coupling $(\Delta / \lambda$ $>1$ ) and for slow rocking motion. These trends are seen for all three relative orientations, the most noticeable differences being that $\Delta \Phi_{\mathrm{S}}$ is least sensitive to $\Delta / \lambda$ for the $Z$ orientation (c) and least sensitive to $k_{\mathrm{r}}$ for the $X$ orientation (a). The larger of the two rocking angles generally gives a smaller anisotropy.

These results can be put into context by considering two extremes. For a $\left[\mathrm{FADH}^{\bullet} \mathrm{Z}^{\bullet}\right]$ pair, in which the static $\mathrm{Z}^{\bullet}$ radical has no orbital angular momentum and no hyperfine couplings, the anisotropy of the reaction yield was separately calculated as $\Delta \Phi_{S}=0.0564$. As expected, precisely the same value is found for [FADH ${ }^{\bullet} \mathrm{O}_{2}^{\bullet-}$ ] when $\mathrm{O}_{2}^{\bullet-}$ is stationary (i.e., $k_{\mathrm{r}}=\beta=0$ ) and its orbital angular momentum is completely quenched $(\Delta / \lambda \gg 1)$. The largest values of $\Delta \Phi_{\mathrm{S}}$ in Fig. 3 (when $k_{\mathrm{r}}=10^{6} \mathrm{~s}^{-1}$ and $\Delta / \lambda=10$ ) are close to this value. The opposite extreme would be a $\mathrm{O}_{2}^{\circ-}$ radical with no
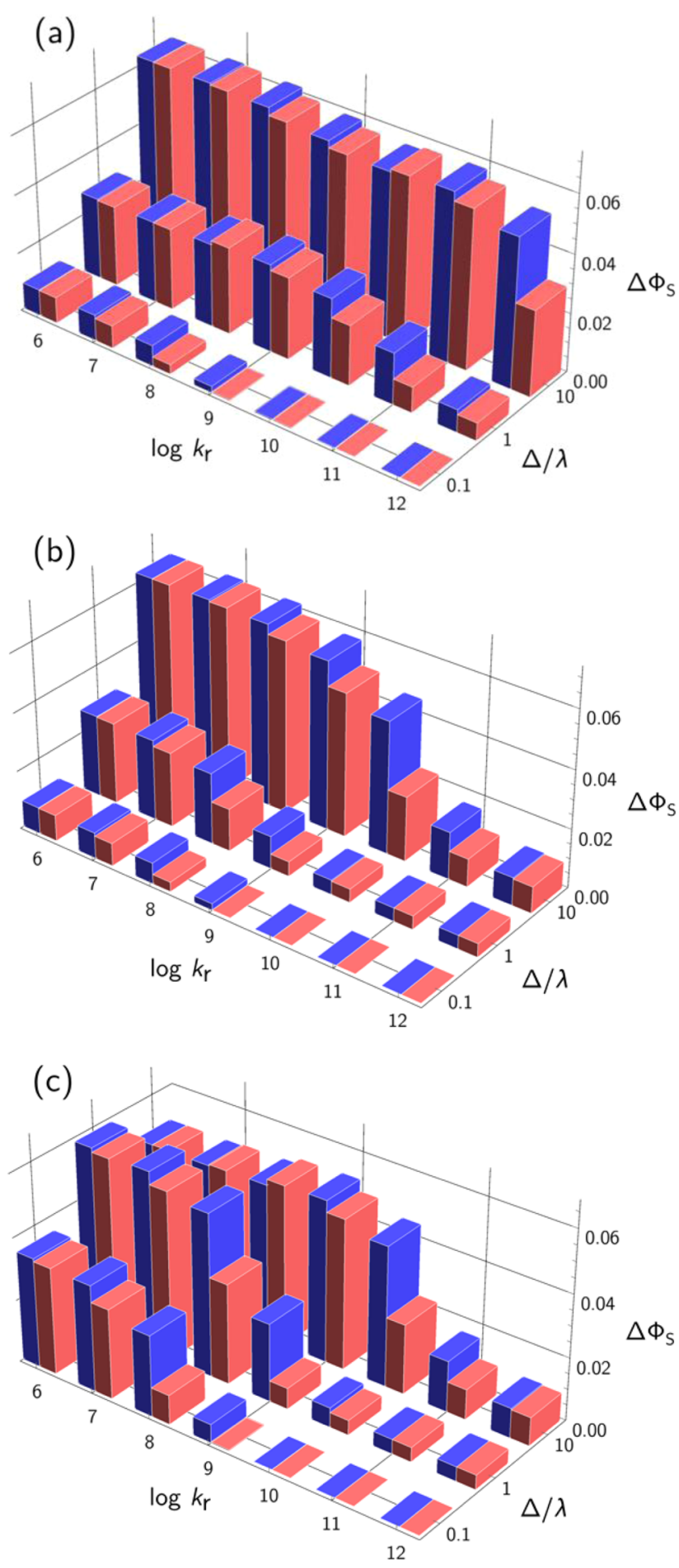

FIG. 3. Singlet yield anisotropy $\Delta \Phi_{\mathrm{S}}$ as a function of the rocking rate constant $k_{\mathrm{r}}$ and the ratio of the ligand field splitting, $\Delta$, to the spin-orbit coupling, $\lambda$, for a model [FADH $\mathrm{O}_{2}^{\bullet-}$ ] radical pair. The $\mathrm{FADH}{ }^{\bullet}$ radical is oriented such that its $z$ axis is parallel to (a) the $X$-axis, (b) the $Y$-axis, and (c) the $Z$-axis (see Fig. 2 for the definition of axis systems). Blue and red correspond to $\beta=2^{\circ}$ and $5^{\circ}$, respectively. In all calculations we have assumed that $\Delta<0$ so that $\Delta / \lambda>0$. 
ligand field splitting, tumbling with a rotational correlation time, $\tau_{\mathrm{c}}$, appropriate for $\mathrm{O}_{2}^{\bullet-}$ in water at physiological temperature. Taking $\eta=6.9 \times 10^{-4} \mathrm{~kg} \mathrm{~m}^{-1} \mathrm{~s}^{-1}$ for the viscosity of water at $310 \mathrm{~K}^{58}$ and a hydrodynamic radius of $150 \mathrm{pm}$, the Stokes-Einstein equation, $\tau_{\mathrm{c}}=4 \pi \eta a^{3} / 3 k_{\mathrm{B}} T$, gives $\tau_{\mathrm{c}}=2.3 \mathrm{ps}$, which is close to the $2.5 \mathrm{ps}$ estimate used by Karogodina et al. ${ }^{42}$ The nearest the data in Fig. 3 come to this limit is when $k_{\mathrm{r}}=10^{12} \mathrm{~s}^{-1}$ and $\Delta / \lambda=0.1$, conditions in which $\Delta \Phi_{\mathrm{S}} \approx 0$

To function as a geomagnetic compass sensor, a radical pair would need to give rise to strongly anisotropic reaction product yields. When one of the radicals is $\mathrm{O}_{2}^{\bullet-}$, this can only occur when its rotational motion is slow $\left(k_{\mathrm{r}} \leq 10^{9} \mathrm{~s}^{-1}\right)$ and its ligand field splitting large $(\Delta / \lambda \geq 1)$.

\section{DISCUSSION}

The conclusion we draw from Fig. 3 is that three interconnected conditions would have to be satisfied for the M2 scheme (Fig. 1) to be a source of geomagnetic directional information. (a) The local electronic environment of the $\mathrm{O}_{2}^{\bullet-}$ radical must be asymmetric such that the difference in energy of the two $\pi$-orbitals exceeds the spin-orbit coupling, i.e., $|\Delta|>\sim 200 \mathrm{~cm}^{-1} \approx k_{\mathrm{B}} T$. (b) $\mathrm{O}_{2}^{\bullet-}$ must rotate at least 1000 times less rapidly than it does in aqueous solution. (c) $\mathrm{O}_{2}^{\bullet-}$ cannot be free to rotate end-over-end; only low-amplitude librations are acceptable. Two further restrictions, which also apply to $\left[\mathrm{FAD}^{\bullet-} \operatorname{TrpH}^{\bullet+}\right]$, can be identified. First, the $\left[\mathrm{FADH}^{\bullet} \mathrm{O}_{2}^{\bullet-}\right.$ ] reactions shown in Figs. 1(i) and $1(\mathrm{j})$ must both proceed on a $\sim 1 \mu$ s time scale. If they are much faster, there will be no time for a $50 \mu \mathrm{T}$ field to affect the spin dynamics; if they are any slower, spin relaxation in $\mathrm{FADH}^{\bullet}$ (if not in $\mathrm{O}_{2}^{\bullet-}$ ) will destroy the spin coherence. ${ }^{57}$ Second, the electron exchange and dipolar interactions should not be much stronger than the Zeeman $(50 \mu \mathrm{T})$ and hyperfine $(\sim 1 \mathrm{mT})$ interactions; otherwise, there will be no singlet-triplet interconversion. ${ }^{41}$ The net effect of these two additional conditions is that the $\mathrm{O}_{2}^{\bullet-}$ radical should not be closer than $\sim 1.5 \mathrm{~nm}$ from the FAD and probably not much further away than $\sim 2.0 \mathrm{~nm}^{17,23,57}$

To satisfy all of these conditions, it seems clear that $\mathrm{O}_{2}^{\bullet-}$ would have to be tightly bound to the protein, presumably via hydrogen bonding. There seems to be little hard experimental evidence for a specific binding site for either $\mathrm{O}_{2}^{\bullet-}$ or $\mathrm{O}_{2}$ in a cryptochrome. Molecular dynamics simulations and electronic structure calculations, however, suggest that $\mathrm{O}_{2}^{\bullet-}$ and its protonated form, $\mathrm{HO}_{2}^{\bullet}$, can be stabilized by hydrogen bonding to reduced forms of FAD in Drosophila melanogaster cryptochrome. ${ }^{59}$ Both radicals were found $\sim 0.3 \mathrm{~nm}$ from one of the ring carbon atoms of the flavin group and a $\sim 40 \mathrm{meV}$ singlet-triplet splitting was calculated for $\left[\mathrm{FAD}^{\bullet-} \mathrm{HO}_{2}^{\bullet}\right.$ ]. Despite the hydrogen bonding, the rotational motion of the radicals does not appear to be highly constrained in either amplitude or frequency. Contrary to the conclusion of that report, ${ }^{59}$ such radical pairs are not suitable as geomagnetic sensors. The electronic exchange interaction $(\sim 40 \mathrm{meV})$, expressed in magnetic field units, is $345 \mathrm{~T}$, i.e., $7 \times 10^{6}$ times stronger than the geomagnetic field and $10^{5}$ times stronger than the hyperfine interactions. Such an enormous singlet-triplet energy gap would prevent the development of any magnetic field effect whether or not conditions (a)-(c) above are satisfied. Identification of $\mathrm{O}_{2}^{\bullet-}$ binding site $>1.5 \mathrm{~nm}$ away from the flavin, with an exchange coupling smaller than the hyperfine interactions (i.e., $<100 \mathrm{neV}$ ) would lend some credence to the superoxide hypothesis.

The case for the involvement of $\mathrm{O}_{2}^{\bullet-}$ in magnetic sensing in vivo would be strengthened if it could be shown convincingly that the rate of oxidation of $\mathrm{FADH}^{-}$in cryptochrome is altered by an applied magnetic field in an oxygen-dependent manner. Further support would come from a strong magnetic isotope effect on the oxidation rate. Substitution of ${ }^{17} \mathrm{O}_{2}$ for ${ }^{16} \mathrm{O}_{2}$ would introduce hyperfine interactions, which should strongly attenuate any magnetic field effect and also accelerate the formation of $\mathrm{FAD}^{\mathrm{ox}}$ (see the Appendix). Given the stringent conditions listed above, the involvement of superoxide in compass magnetoreception in migratory songbirds must remain highly speculative until such experimental evidence is forthcoming.

\section{ACKNOWLEDGMENTS}

This work was supported by the Air Force Office of Scientific Research (Air Force Materiel Command, USAF Award No. FA9550-14-1-0095), the European Research Council (under the European Union's Horizon 2020 research and innovation program, Grant Agreement No. 810002, Synergy Grant, QuantumBirds), and Deutsche Forschungsgemeinschaft SFB 1372 "Magnetoreception and Navigation in Vertebrates." We are grateful to Thomas Fay for calculating the hyperfine coupling tensors of ${ }^{17} \mathrm{O}_{2}^{\bullet-}$ and to Jiwang Chen for performing initial calculations on the magnetic sensitivity of ${ }^{17} \mathrm{O}_{2}^{\bullet-}$-containing radical pairs.

\section{APPENDIX: ${ }^{17}$ O MAGNETIC ISOTOPE EFFECT}

${ }^{17} \mathrm{O}$ hyperfine tensors for ${ }^{17} \mathrm{O}_{2}^{\bullet-}$ were calculated using Gaussian 09 with B3LYP/6-311++G(d,p) for geometry optimization and B3LYP/EPR-III for the hyperfine interactions. Implicit solvation (water) was included via the polarizable continuum model. The isotropic and anisotropic components of the hyperfine tensors of both ${ }^{17} \mathrm{O}$ atoms were $-1.508 \mathrm{mT}$ and $(3.1372,3.0015,-6.1387)$ $\mathrm{mT}$, respectively. $\Delta \Phi_{\mathrm{S}}$ was calculated using a similar method to that described above, with two identical spin-5/2 nuclear spins in the superoxide radical and the above hyperfine couplings. Rocking motion of the ${ }^{17} \mathrm{O}_{2}^{\bullet-}$ radical was not included $\left(k_{\mathrm{r}}=\beta=0\right)$. The results are shown in Table I as the ratio of $\Delta \Phi_{\mathrm{S}}$ for $\left[\mathrm{FADH}^{\bullet}{ }^{16} \mathrm{O}_{2}^{\bullet-}\right.$ ] to that for $\left[\mathrm{FADH}^{\bullet}{ }^{17} \mathrm{O}_{2}^{\bullet-}\right.$ ] for three values of $\Delta / \lambda$ and the three relative orientations of $\mathrm{FADH}^{\bullet}$ and $\mathrm{O}_{2}^{\bullet-}$ considered above. Inclusion of the ${ }^{17} \mathrm{O}$ hyperfine interactions causes a 4 - to 10 -fold reduction in the anisotropy of the magnetic field effect for $\Delta / \lambda=1.0$ and 10.0. The change is less pronounced for $\Delta / \lambda=0.1$, where the values of $\Delta \Phi_{\mathrm{S}}$ are much smaller.

TABLE I. Ratio of reaction yields anisotropies for ${ }^{16} \mathrm{O}_{2}^{\bullet-}$ and ${ }^{17} \mathrm{O}_{2}^{\bullet-}$ containing radical pairs.

\begin{tabular}{cccc}
\hline \hline & $\Delta / \lambda=0.1$ & $\Delta / \lambda=1.0$ & $\Delta / \lambda=10.0$ \\
\hline$X$ & 1.00 & 3.84 & 10.11 \\
$Y$ & 1.00 & 3.78 & 8.49 \\
$Z$ & 2.31 & 3.73 & 3.54 \\
\hline \hline
\end{tabular}




\section{REFERENCES}

${ }^{1}$ W. Wiltschko and R. Wiltschko, Science 176, 62 (1972).

${ }^{2}$ G. C. Nordmann, T. Hochstoeger, and D. A. Keays, PLoS Biol. 15, e2003234 (2017).

${ }^{3}$ H. Mouritsen, Nature 558, 50 (2018).

${ }^{4}$ S. Akesson, J. Morin, R. Muheim, and U. Ottosson, Proc. R. Soc. London, Ser. B 268, 1907 (2001).

${ }^{5}$ N. Lefeldt, D. Dreyer, N. L. Schneider, F. Steenken, and H. Mouritsen, J. Exp. Biol. 218, 206 (2015).

${ }^{6}$ J. L. Kirschvink and J. L. Gould, Biosystems 13, 181 (1981).

${ }^{7}$ J. L. Kirschvink, M. M. Walker, and C. E. Diebel, Curr. Opin. Neurobiol. 11, 462 (2001).

${ }^{8}$ M. Winklhofer and J. L. Kirschvink, J. R. Soc., Interface 7, S273 (2010).

${ }^{9}$ P. J. Hore and H. Mouritsen, Annu. Rev. Biophys. 45, 299 (2016).

${ }^{10}$ K. Schulten, C. E. Swenberg, and A. Weller, Z. Phys. Chem. 111, 1 (1978).

${ }^{11}$ T. Ritz, S. Adem, and K. Schulten, Biophys. J. 78, 707 (2000).

${ }^{12}$ M. Ahmad and A. R. Cashmore, Nature 366, 162 (1993).

${ }^{13}$ M. Liedvogel and H. Mouritsen, J. R. Soc., Interface 7, S147 (2010).

${ }^{14}$ H. Mouritsen and P. J. Hore, Curr. Opin. Neurobiol. 22, 343 (2012).

${ }^{15}$ C. A. Dodson, P. J. Hore, and M. I. Wallace, Trends Biochem. Sci. 38, 435 (2013).

${ }^{16}$ Y. T. Kao, C. Tan, S. H. Song, N. Ozturk, J. Li, L. J. Wang, A. Sancar, and D. P. Zhong, J. Am. Chem. Soc. 130, 7695 (2008).

${ }^{17}$ P. Müller, J. Yamamoto, R. Martin, S. Iwai, and K. Brettel, Chem. Commun. 51, 15502 (2015).

${ }^{18}$ D. Nohr, S. Franz, R. Rodriguez, B. Paulus, L.-O. Essen, S. Weber, and E. Schleicher, Biophys. J. 111, 301 (2016).

${ }^{19}$ K. B. Henbest, K. Maeda, P. J. Hore, M. Joshi, A. Bacher, R. Bittl, S. Weber, C. R. Timmel, and E. Schleicher, Proc. Natl. Acad. Sci. U. S. A. 105, 14395 (2008).

${ }^{20}$ K. Maeda, A. J. Robinson, K. B. Henbest, H. J. Hogben, T. Biskup, M. Ahmad, E. Schleicher, S. Weber, C. R. Timmel, and P. J. Hore, Proc. Natl. Acad. Sci. U. S. A. 109, 4774 (2012).

${ }^{21}$ H. Mouritsen, D. Heyers, and O. Güntürkün, Annu. Rev. Physiol. 78, 133 (2016).

${ }^{22}$ H. G. Hiscock, T. W. Hiscock, D. R. Kattnig, T. Scrivener, A. M. Lewis, D. E. Manolopoulos, and P. J. Hore, Q. Rev. Biophys. 52, e9 (2019).

${ }^{23}$ D. M. W. Sheppard, J. Li, K. B. Henbest, S. R. T. Neil, K. Maeda, J. Storey, E. Schleicher, T. Biskup, R. Rodriguez, S. Weber, P. J. Hore, C. R. Timmel, and S. R. Mackenzie, Sci. Rep. 7, 42228 (2017).

${ }^{24}$ H. J. Hogben, O. Efimova, N. Wagner-Rundell, C. R. Timmel, and P. J. Hore, Chem. Phys. Lett. 480, 118 (2009).

${ }^{25}$ I. A. Solov'yov and K. Schulten, Biophys. J. 96, 4804 (2009).

${ }^{26}$ P. Müller and M. Ahmad, J. Biol. Chem. 286, 21033 (2011).

${ }^{27}$ B. D. Zoltowski, Y. Chelliah, A. Wickramaratne, L. Jarocha, N. Karki, W. Xu, H. Mouritsen, P. J. Hore, R. E. Hibbs, C. B. Green, and J. S. Takahashi, Proc. Natl. Acad. Sci. U. S. A. 116, 19449 (2019).

${ }^{28}$ A. A. Lee, J. C. S. Lau, H. J. Hogben, T. Biskup, D. R. Kattnig, and P. J. Hore, J. R. Soc., Interface 11, 20131063 (2014).

${ }^{29}$ S. Schwarze, N.-L. Schneider, T. Reichl, D. Dreyer, N. Lefeldt, S. Engels, N. Baker, P. J. Hore, and H. Mouritsen, Front. Behav. Neurosci. 10, 55 (2016).
${ }^{30}$ S. Engels, N. L. Schneider, N. Lefeldt, C. M. Hein, M. Zapka, A. Michalik, D. Elbers, A. Kittel, P. J. Hore, and H. Mouritsen, Nature 509, 353 (2014).

${ }^{31}$ H. G. Hiscock, H. Mouritsen, D. E. Manolopoulos, and P. J. Hore, Biophys. J. 113, 1475 (2017).

${ }^{32}$ V. Massey, J. Biol. Chem. 269, 22459 (1994).

${ }^{33}$ L. J. G. W. van Wilderen, G. Silkstone, M. Mason, J. J. van Thor, and M. T. Wilson, FEBS Open Bio 5, 885 (2015).

${ }^{34}$ C. Niessner, S. Denzau, K. Stapput, M. Ahmad, L. Peichl, W. Wiltschko, and R. Wiltschko, J. R. Soc., Interface 10, 20130638 (2013).

${ }^{35}$ C. Niessner, S. Denzau, L. Peichl, W. Wiltschko, and R. Wiltschko, J. Exp. Biol. 217, 4221 (2014).

${ }^{36}$ C. Niessner, S. Denzau, L. Peichl, W. Wiltschko, and R. Wiltschko, J. Comp. Physiol., A 204, 977 (2018).

${ }^{37}$ R. Wiltschko, M. Ahmad, C. Niessner, D. Gehring, and W. Wiltschko, J. R. Soc., Interface 13, 20151010 (2016).

${ }^{38}$ R. Wiltschko and W. Wiltschko, J. R. Soc., Interface 16, 20190295 (2019).

${ }^{39}$ M. Pooam, L. D. Arthaut, D. Burdick, J. Link, C. F. Martino, and M. Ahmad, Planta 249, 319 (2019).

${ }^{40}$ C. T. Rodgers, S. A. Norman, K. B. Henbest, C. R. Timmel, and P. J. Hore, J. Am. Chem. Soc. 129, 6746 (2007).

${ }^{41}$ O. Efimova and P. J. Hore, Biophys. J. 94, 1565 (2008).

${ }^{42}$ T. Y. Karogodina, I. G. Dranov, S. V. Sergeeva, D. V. Stass, and U. E. Steiner, Chem. Phys. Chem. 12, 1714 (2011).

${ }^{43}$ S. Worster, D. R. Kattnig, and P. J. Hore, J. Chem. Phys. 145, 035104 (2016).

${ }^{44}$ D. R. Kattnig, J. K. Sowa, I. A. Solov'yov, and P. J. Hore, New J. Phys. 18, 063007 (2016).

${ }^{45}$ D. R. Kattnig, I. A. Solov'yov, and P. J. Hore, Phys. Chem. Chem. Phys. 18, 12443 (2016).

${ }^{46}$ K. P. Huber and G. Herzberg, Molecular Spectra and Molecular Structure (Van Nostrand Reinhold, New York, 1979).

${ }^{47}$ D. R. Kattnig, J. Phys. Chem. B 121, 10215 (2017).

${ }^{48}$ T. Y. Karogodina, S. V. Sergeeva, and D. V. Stass, Appl. Magn. Reson. 36, 195 (2009).

${ }^{49}$ M. C. R. Symons, G. W. Eastland, and L. R. Denny, J. Chem. Soc., Faraday Trans. 1 76, 1868 (1980).

${ }^{50}$ W. Kanzig and M. H. Cohen, Phys. Rev. Lett. 3, 509 (1959).

${ }^{51}$ R. J. Usselman, I. Hill, D. J. Singel, and C. F. Martino, PLoS One 9, e93065 (2014).

${ }^{52}$ R. J. Usselman, C. Chavarriaga, P. R. Castello, M. Procopio, T. Ritz, E. A. Dratz, D. J. Singel, and C. F. Martino, Sci. Rep. 6, 38543 (2016).

${ }^{53}$ D. R. Kattnig and P. J. Hore, Sci. Rep. 7, 11640 (2017).

${ }^{54}$ F. Cintolesi, T. Ritz, C. W. M. Kay, C. R. Timmel, and P. J. Hore, Chem. Phys. 294, 385 (2003).

${ }^{55}$ H. G. Hiscock, S. Worster, D. R. Kattnig, C. Steers, Y. Jin, D. E. Manolopoulos, H. Mouritsen, and P. J. Hore, Proc. Natl. Acad. Sci. U. S. A. 113, 4634 (2016).

${ }^{56}$ H. R. Zeller, R. T. Shuey, and W. Känzig, J. Phys. Colloq. 28, C4-81 (1967).

${ }^{57}$ C. T. Rodgers and P. J. Hore, Proc. Natl. Acad. Sci. U. S. A. 106, 353 (2009).

${ }^{58}$ L. Korson, W. Drosthan, and F. J. Millero, J. Phys. Chem. 73, 34 (1969).

${ }^{59}$ P. Mondal and M. Huix-Rotllant, Phys. Chem. Chem. Phys. 21, 8874 (2019). 